\title{
Peripapillary hyperreflective ovoid mass-like structures (PHOMS): OCTA may reveal new findings
}

\author{
Enrico Borrelli $\mathbb{1}^{1}$ - Piero Barboni ${ }^{1,2}$ - Marco Battista ${ }^{1} \cdot$ Riccardo Sacconi $\mathbb{i}^{1} \cdot$ Lea Querques $^{1}$. \\ Maria Lucia Cascavilla ${ }^{1}$. Francesco Bandello ${ }^{1} \cdot$ Giuseppe Querques $^{1}$
}

Received: 15 January 2020 / Revised: 13 February 2020 / Accepted: 9 April 2020 / Published online: 28 April 2020

(c) The Author(s), under exclusive licence to The Royal College of Ophthalmologists 2020

\begin{abstract}
Purpose Peripapillary hyperreflective ovoid mass-like structures (PHOMS) are novel and not well characterized findings occurring in several disorders of the optic nerve. The aim of this study is to present two cases of tilted disc syndrome (TDS) and one case with optic disc drusen undergoing a multimodal imaging approach.

Methods In this case series, a qualitative evaluation of the OCTA findings in regions with PHOMS was performed.

Results Structural OCT revealed the presence of PHOMS. OCTA identified the presence of a vascular complex within this hyperreflective structure.

Conclusions Assuming that PHOMS are thought to correspond to herniating nerve fibers or be secondary to axoplasmic stasis, this vascular complex may represent a displacement of the deeper vessels deputed at the irroration of the optic nerve into the retina or, alternatively, might be secondary to an increase in vascular endothelial growth factor (VEGF) levels and a subsequent development of neovessels.
\end{abstract}

\section{Introduction}

Tilted disc syndrome (TDS) is a congenital anomaly occurring in $1-2 \%$ of the population $[1,2]$. This disease is secondary to an incomplete closure of the fetal fissure of the eye, this causing the optic nerve penetrating the eye with an oblique angle [1, 2]. An inferonasal crescent, situs inversus of the retinal vessels, myopic astigmatism, and visual field defect represent other main characteristics of this syndrome [1, 2]. Importantly, TDS has been proved to be associated with high myopia, as this disorder affects one fifth of patients with more than 5 diopters (D) of myopia [1].

Optic disc drusen are acellular calcific deposits which may occur in small optic nerve heads [3]. Important evidences suggest that axoplasmic transport disturbance and axonal degeneration may be involved in the development of

Giuseppe Querques

giuseppe.querques@hotmail.it

1 Department of Ophthalmology, University Vita-Salute, IRCCS Ospedale San Raffaele, Milan, Italy

2 Studio Oculistico d'Azeglio, Bologna, Italy these alterations [4]. There is a large variability in number and size of optic disc drusen, and they may be visible near the optic disc surface or buried within the optic nerve head, provoking the development of a pseudopapilledema [5].

Peripapillary hyperreflective ovoid mass-like structures (PHOMS) represent an optical coherence tomography (OCT) finding which has been described in several disorder, including papilledema [6], TDS [7], optic disc drusen [8], and other forms of pseudopapilledema [6]. Despite a rising debate about their actual origin $[9,10]$, there is an increasing evidence suggesting that PHOMS may correspond to herniating nerve fibers $[7,8,10]$.

In this case series, we provided an integrate multimodal imaging description of PHOMS is two cases with tilted disc syndrome and one case with optic disc drusen. In details, OCT angiography (OCTA) findings may suggest the presence of a complex vascular structure within this hyperreflective peripapillary material.

\section{Methods}

All patients in this study consented to the presentation of deidentified information pertaining to their pathology. The study was approved by the San Raffaele IRB and adhered to 

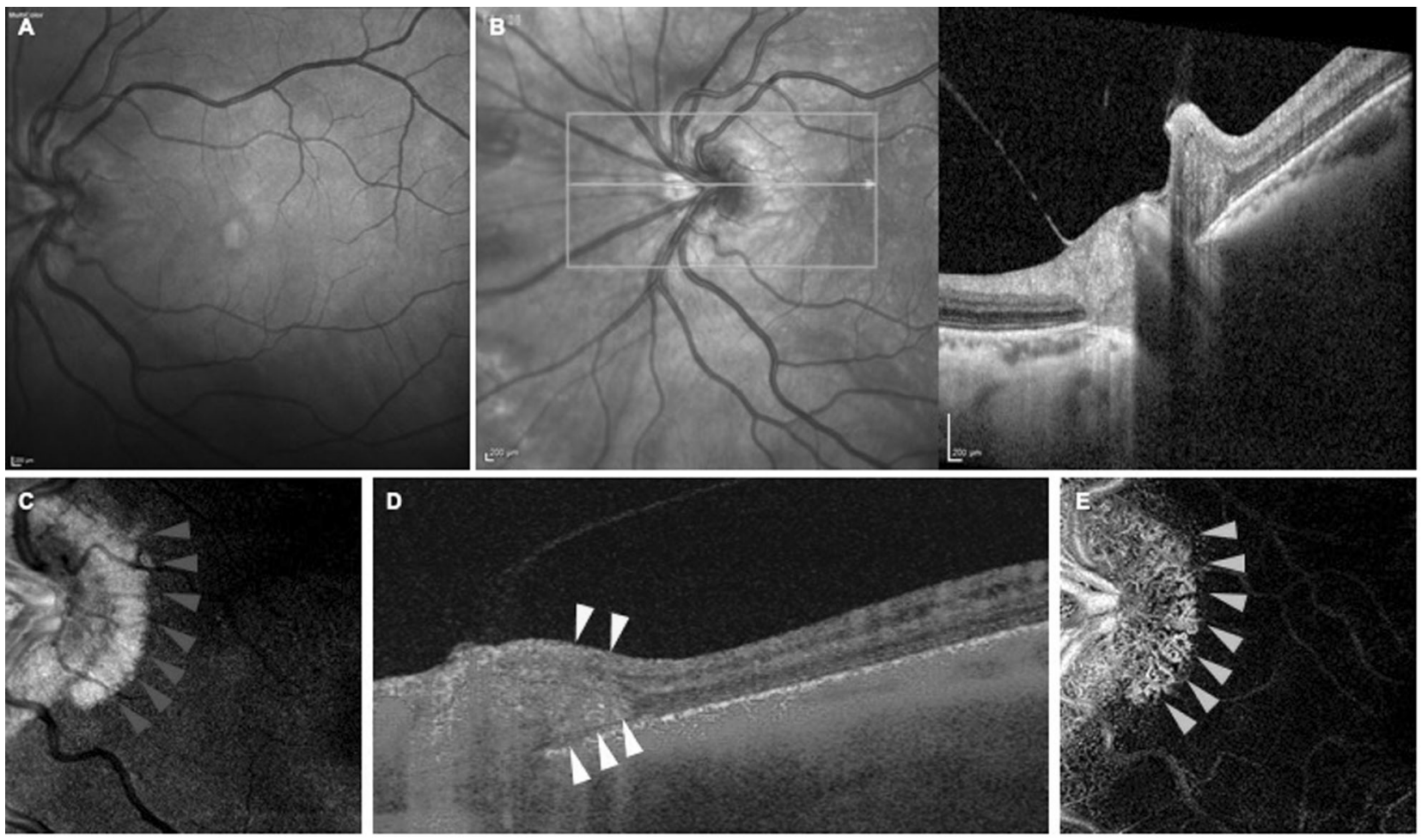

Fig. 1 Multimodal imaging from the case \#1. a Multicolor image confirmed presence of tilted disc syndrome. b Structural OCT identifies the presence of a peripapillary hyperreflective ovoid mass-like structure (PHOMS) (this scan was at the level of the green arrow on the corresponding near infrared image). $\mathbf{c}$ The en face structural OCT image including this hyperreflective structure allowed to identify the topographical broadening of this lesion (highlighted with blue arrow heads) which was mainly located temporally and superiorly to the optic nerve. d The corresponding OCTA B-scan displays the presence of blood flow within this hyperreflective lesion (white arrow heads). This flow is separated by that corresponding to the more superficial radial peripapillary complex (yellow arrow heads). e On the en face OCTA image, a wreath-shaped vascular complex was visualized (green arrow heads). the tenets of the Declaration of Helsinki and Health Insurance Portability and Accountability Act.

Patients underwent a complete ophthalmological examination, which included structural OCT and OCTA imaging.

\section{Results}

\section{Case 1}

A 35-year-old woman was referred to our department complaining of reduced visual acuity in the left eye (LE). The patient had high myopia in both eyes $(-13.50 \mathrm{D}$ and $-4.50 \mathrm{D}$ in the right $[\mathrm{RE}]$ and LE, respectively). Her medical history was otherwise unremarkable. Bestcorrected visual acuity (BCVA) was 20/20 Snellen in the RE and 20/25 in the LE. Slit-lamp anterior segment examination and intraocular pressure were normal. Fundoscopy revealed a tessellated appearance of the fundus in both eyes. Furthermore, tilted disc accompanied by situs inversus of the retinal vessels and thinning of the nasal retinal pigment epithelium and choroid was revealed in the LE. The patient also underwent swept source
(SS)-OCT structural and angiography examination. Peripapillary structural SS-OCT B-scan revealed the presence of an hyperreflective ovoid mass-like structure in the LE, which was located in the temporal sector. SS-OCTA B-scan of the same area displayed the presence of flow within this hyperreflective region. En face SS-OCTA analysis allowed the visualization of a wreathshaped vascular complex localized in the same region (Fig. 1).

\section{Case 2}

A 54-year-old woman was referred to our department presented with reduced visual acuity in the left eye (LE). The patient had slight myopia in both eyes $(-0.50 \mathrm{D}$ in the $\mathrm{RE}$ and $-1.75 \mathrm{D}$ in the LE). Her medical history was otherwise unremarkable. At presentation, BCVA was 20/ 25 Snellen in the RE and 20/32 in the LE. Slit-lamp anterior segment examination and intraocular pressure were normal. TDS was revealed in the LE. Peripapillary structural SS-OCT B-scans revealed the presence of PHOMS in the LE, which was located in the temporal sector. SS-OCTA B-scan of the same area displayed the 
Fig. 2 Multimodal imaging from the case \#2. a Near infrared image confirmed presence of tilted disc syndrome. b Structural OCT scan at the level of the green arrow displayed the presence of a PHOMS (yellow arrows). c The OCTA B-scan showed the presence of blood flow within this hyperreflective lesion (white arrows). d The en face OCTA image including this hyperreflective structure allowed to identify the expansion of these vessels.
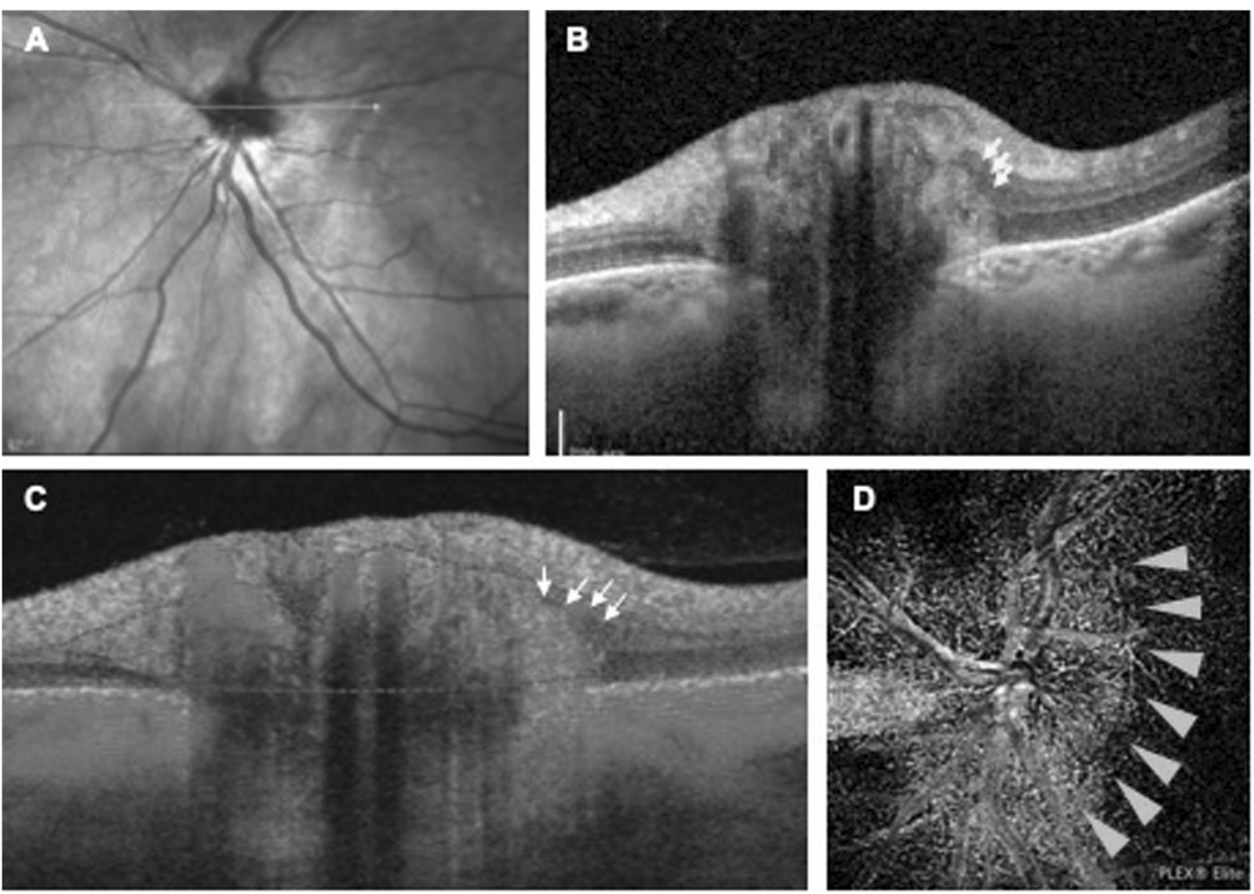

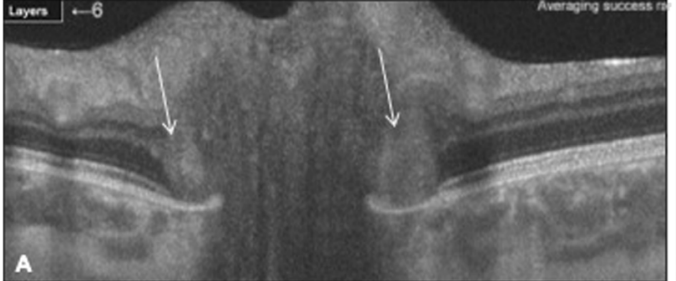

Fig. 3 Multimodal imaging from the case \#3. a Structural OCT identifies the presence of two peripapillary hyperreflective ovoid masslike structures (PHOMS) (yellow arrows). b The OCTA B-scan confirmed the presence of flow signal within these hyperreflective lesions

presence of flow within this hyperreflective region. En face SS-OCTA analysis allowed the visualization of a wreath-shaped vascular complex localized in the same region (Fig. 2).

\section{Case 3}

A 10-year-old boy was referred to our department for a routine examination. At presentation, BCVA was 20/20 in either eye. Slit-lamp anterior segment examination and intraocular pressure were normal. Indirect ophthalmoscopy revealed the presence of pseudopapilledema in the $\mathrm{RE}$ because of the presence of optic disc drusen, as revealed by a multimodal imaging approach. Peripapillary structural SS-OCT B-scans revealed the presence of PHOMSs, which were located in the nasal and temporal sectors. SS-OCTA B-scan of the same area displayed the presence of flow within these hyperreflective regions (Fig. 3). (white arrows). c The en face OCTA image including this hyperreflective structure granted the topographical identification of these vessels (green arrow heads).

\section{Discussion}

In this series we reported an OCTA description of PHOMS in two cases with TDS and one case with optic disc drusen. Overall, we observed that this hyperreflective lesion may include a vascular complex and its composition is thus more complicated than previously thought.

Several previous studies employing structural OCT have furnished a detailed description of eyes with TDS. Shinohara et al. [2] investigated 54 TDS eyes of 36 adult subjects, while Pichi and colleagues [7] enrolled 38 eyes of 20 pediatric patients with TDS. In both studies, the OCT images obtained in the peripapillary region displayed a protrusion of the upper edge of Bruch's membrane and choroid toward the disc area in a percentage of the enrolled eyes. More importantly, this protrusion was associated with the presence of co-localizing PHOMS.

Although they may coexist with optic disc drusen and differential diagnosis may be challenging because of their 
oval shape, PHOMS have some important peculiarities which may help in their identification: $[8,11]$ (i) on OCT images they are hyperreflective lesions without an hyporeflective core; (ii) they often localize adjacent to regions of pseudopapilledema; (iii) they are not characterized by an increased autofluorescence; (iv) despite their superficial location, PHOMS are not visible on ultrasound B-scan.

Several histopathological evidences suggest that PHOMS represent lateral bulges of nerve fibers $[8,12,13]$. Furthermore, previous studies argued that these fibers may have herniated above Bruch's membrane $[2,7,8]$ or, alternatively, they could represent obstructed axoplasmic material $[8,11]$.

In recent years, OCTA technology has been revealing for both normal and diseased eyes and has significantly expanded the assessment of the deeper vascular layers, given the limitations of previous dye-based imaging techniques [14]. In this report, we employed OCTA to examine three patients with PHOMS. We showed that these hyperreflective lesions may contain a vascular complex which seems not to be in continuity with the physiological vasculature located in the peripapillary region. There are several possible explanations for the presence of vessels within PHOMS. A herniation of nerve fibers might bring deeper vessels deputed at the irroration of the optic nerve into the retina. Alternatively, distended or displaced axons, axoplasmic stasis, or congestion in the prelaminar optic nerve head might cause an increase in vascular endothelial growth factor (VEGF) levels and a subsequent development of neovessels.

In conclusion, a multimodal imaging strategy proved to be an optimal method for a better characterization of PHOMS occurring in eyes with tilted disc or optic disc drusen. OCTA imaging revealed that these hyperreflective lesions may contain a vascular complex. Future larger longitudinal studies may reveal the exact mechanisms leading to the formation of these vessels.

\section{What was known before}

- Peripapillary hyperreflective ovoid mass-like structures (PHOMS) are novel and not well characterized findings occurring in several disorders of the optic nerve.

- PHOMS are thought to correspond to herniating nerve fibers or to be secondary to axoplasmic stasis.

\section{What this study adds}

- OCTA identified the presence of a vascular complex within PHOMS.
- This vascular complex may represent a displacement of physiological deeper vessels or, alternatively, it may be secondary to neovessels.

\section{Compliance with ethical standards}

Conflict of interest The authors declare that they have no conflict of interest.

Publisher's note Springer Nature remains neutral with regard to jurisdictional claims in published maps and institutional affiliations.

\section{References}

1. Ohno-Matsui K. Pathologic myopia. Asia-Pacific J Ophthalmol. 2016;5:415-23.

2. Shinohara K, Moriyama M, Shimada N, Nagaoka N, Ishibashi T, Tokoro T, et al. Analyses of shape of eyes and structure of optic nerves in eyes with tilted disc syndrome by swept-source optical coherence tomography and three-dimensional magnetic resonance imaging. Eye. 2013;27:1233-42.

3. Lam BL, Morais CG, Pasol J. Drusen of the optic disc. Curr Neurol Neurosci Rep. 2008;8:404.

4. Spencer WH. Drusen of the optic disk and aberrant axoplasmic transport. The XXXIV Edward Jackson memorial lecture. Am J Ophthalmol. 1978;85:1-12.

5. Carta A, Mora P, Aldigeri R, Gozzi F, Favilla S, Tedesco S, et al. Optical coherence tomography is a useful tool in the differentiation between true edema and pseudoedema of the optic disc. PLoS ONE. 29;13:e0208145.

6. Bassi ST, Mohana KP. Optical coherence tomography in papilledema and pseudopapilledema with and without optic nerve head drusen. Indian J Ophthalmol. 2014;62:1146.

7. Pichi F, Romano S, Villani E, Lembo A, Gilardoni F, Morara M, et al. Spectral-domain optical coherence tomography findings in pediatric tilted disc syndrome. Graefe's Arch Clin Exp Ophthalmol. 2014;252:1661-7.

8. Malmqvist L, Bursztyn L, Costello F, Digre K, Fraser JA, Fraser $\mathrm{C}$, et al. The optic disc drusen studies consortium recommendations for diagnosis of optic disc drusen using optical coherence tomography. J Neuro-Ophthalmol. 2018;38:299-307.

9. Lee KM. Peripapillary hyperreflective ovoid mass-like structures: is it optic disc drusen or not? J Neuro-Ophthalmol. 2018;38: $567-8$.

10. Malmqvist L, Bursztyn L, Costello F, Digre K, Fraser JA, Fraser C, et al. Peripapillary hyperreflective ovoid mass-like structures: is it optic disc drusen or not?: response. J Neuro-Ophthalmol. 2018;38:568-70.

11. Wang DD, Leong JCY, Gale J, Wells AP. Multimodal imaging of buried optic nerve head drusen. Eye. 2018;32:1145-6.

12. Sibony PA, Kupersmith MJ. Paton's folds revisited: wrinkles, folds and creases in papilledema. Investig Ophthalmol Vis Sci. 2016;57:4553.

13. Tso MO. Pathology and pathogenesis of drusen of the optic nervehead. Ophthalmology. 1981;88:1066-80.

14. Borrelli E, Sadda SR, Uji A, Querques G. Pearls and pitfalls of optical coherence tomography angiography imaging: a review. Ophthalmol Ther. 2019;8:215-26. 